\title{
Subharmonically injection-locked oscillator using a nonlinear transmission line
}

\author{
Elena Fernández, Mabel Pontón, Almudena Suárez, Franco Ramírez \\ Communications Engineering Department \\ University of Cantabria \\ Santander, Spain
}

\begin{abstract}
This work describes a first realization of an oscillator driven through a nonlinear transmission line (NLTL). In this way, the oscillator is able to synchronize to a sinusoidal injection source of much lower frequency. This can be understood as the result of two processes: high harmonic generation in the NLTL and frequency multiplication plus mixing in the oscillator device. The concept can be applicable in ultrawideband signal oscillators where synchronization should enable a fast oscillation start-up and facilitate the modulation process. As an example, it has been used here to obtain a pulsedenvelope oscillator at $6 \mathrm{GHz}$ injected with a sinusoidal signal in the order of $100 \mathrm{MHz}$. The operation bands have been analyzed with a Poincaré map technique, allowing the detection of the bifurcation phenomena that delimit the stable synchronization ranges. For an understanding of the phase-noise spectrum, this spectrum is analyzed at higher injection frequencies with the conversion matrix approach. Very good results have been obtained in comparison with measurements.
\end{abstract}

Keywords - Injection-locked oscillator, nonlinear transmission line, stability, phase noise

\section{INTRODUCTION}

The synchronization of an oscillator at the frequency $f_{o}$ to low noise sinusoidal source at a subharmonic frequency $f_{o} / N$ provides a compact and flexible mechanism for phase noise reduction of the oscillator signal [1]. However, sub-harmonic injection locking is typically limited to small values of the subharmonic order $N$. This is because when this order increases, synchronization bands decrease and become negligible from certain $N$. Here the possibility to achieve synchronization at high subharmonic order using a nonlinear transmission line (NLTL) will be investigated. NLTLs, usually composed by a high number of inductance-varactor cells [24], enable high harmonic generation and pulse shaping, and can be used in sampling-instrumentation and ultrawideband sensors, among other applications. Here the NLTL will be introduced in the oscillator input network to achieve a pulsed waveform capable to sub-synchronize the oscillator circuit. The NLTL provides high harmonic frequencies, further multiplied due to the nonlinearity behaviour of the active device in the oscillator circuit. Spectral lines in the order of the oscillation frequency should be able to synchronize the oscillation through a process of mixing and feedback [1]. The high harmonic content of the input signal will give rise to several synchronization bandwidths due to a multi-resonance phenomenon [5].
An interesting application of subharmonic injection locking was described in [6-7], where a pulsed injection-locked oscillator is proposed for the generation of single carrier ultrawideband signals. The injection locking allows a fast start-up since the oscillation does not start on the noise but on a harmonic component generated by the pulse [6,7]. The work [5] presented a detailed stability analysis of pulsed-injectionlocked oscillators, demonstrating relatively wide ranges of stable behavior when the oscillator was injected with pulses generated with laboratory instruments. Here the compact NLTL-based oscillator will be driven with an ordinary sinusoidal signal. For a comparison of performance, a properly designed NLTL will be introduced into the driving network of the same oscillator circuit at $6 \mathrm{GHz}$ considered in [5]. The synhcronization bands will be analyzed with a Poincaré map technique, enabling the determination of the bifurcation phenomena that delimit the stable operation ranges. For an understanding of the phase noise behaviour, a higher driving frequency will be considered, analyzing the phase noise spectrum with the conversion matrix approach. The prototype has been manufactured and measured obtaining good agreement between simulated and experimental results.

\section{DESIGN OF THE NLTL BASED SUBINJECTION LOCKED OSCILLATOR}

The subharmonic injection-locked oscillator will be based on the circuit in Fig. 1a, which oscillates when resistor $R_{1}$ is connected to a dc source voltage fulfilling $V_{b}>1$. No oscillation is obtained for bias voltage below this value. The oscillation frequency is $f_{o}=6 \mathrm{GHz}$ and the output signal is extracted at the collector terminal. In [5], the bias source was replaced with a pulse generator having excursions above the threshold voltage $V_{b}$ and therefore switching the oscillation on and off [6-7]. Here the NLTL output must be able to switch the oscillator in a manner similar to the pulse generator in [5]. On the other hand, to enable the synchronized behavior the NLTL must provide high harmonic content. Assuming a sinusoidal input source of low frequency $f_{i n}$, the NLTL will generate the harmonic terms $p \cdot f_{\text {in }}$ where $p$ is an integer. When connected to the NLTL output, the oscillator active device (in non linear operation) will provide higher harmonic frequencies $p \cdot m f_{i n}$, where $m$ is an integer, so that spectral components near the oscillation frequency are able to synchronize the oscillator. There can be several combinations of $p, m$ values. For instance, assuming $f_{\text {in }}=200 \mathrm{MHz}$, and multiplier capability of the active device up to third order, one of these combinations may correspond to 
$p=10$ and $m=3$ and another one to $p=15$ and $m=2$. Due to the highly nonlinear behavior of the system with the NLTL interacting with the oscillator circuit, and the fact that oscillation frequency is pulled by the injection source, this estimation is only approximate. In any case, for a low subsynchronization frequency $f_{i n}$, the NLTL should be able to provide harmonic components $p \cdot f_{i n}$, such that $p \geq 10$.

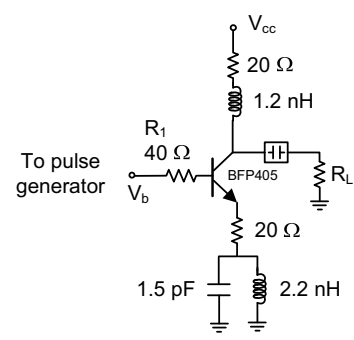

(a)

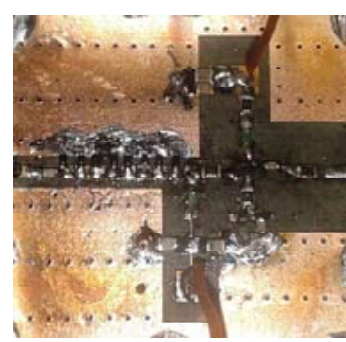

(c)

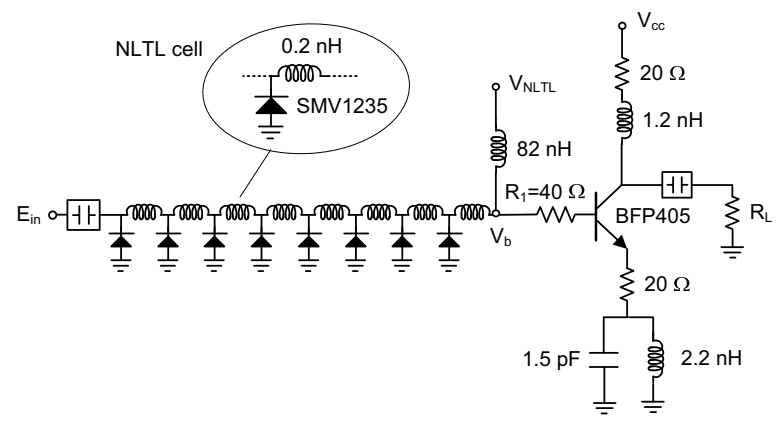

(b)

Fig 1. NLTL based sub-injection locked oscillator. (a) Schematic of the BJTbased oscillator considered in work [5]. (b) Schematic of the proposed design using an NLTL connected at base terminal. (c) Photograph of the compact prototype built on Rogers $4003 \mathrm{C}$ substrate $\left(\varepsilon_{r}=3.38 ; h=0.508 \mathrm{~mm}\right)$, of dimensions $40 \times 40 \mathrm{~mm}^{2}$.

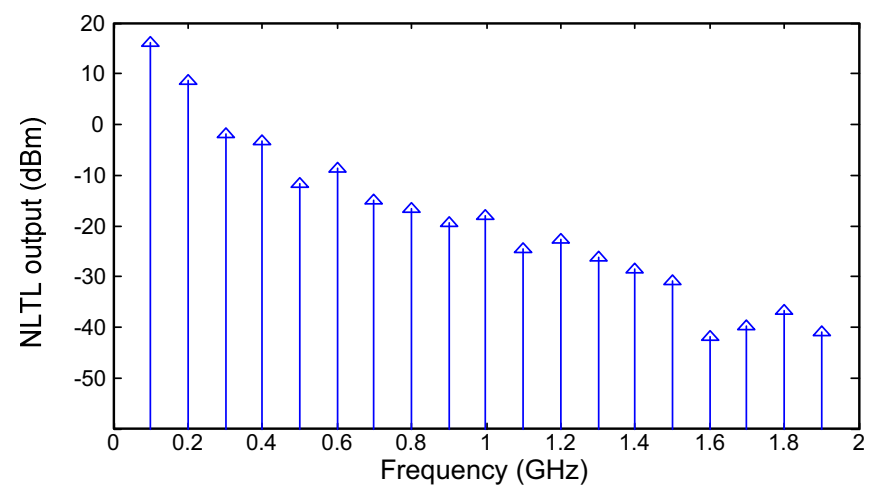

Fig. 2 Output spectrum of the NLTL (simulated separately from the oscillator circuit) when terminated in its characteristic impedance. The input frequency is $f_{\text {in }}=100 \mathrm{MHz}$ and the input amplitude is $E_{\text {in }}=3 \mathrm{~V}$.

Initially, the NLTL has been designed separately from the circuit to optimize its frequency generation capabilities. In the usual case of an NLTL with higher diode cut-off than the Bragg frequency, the latter will limit the maximum input frequency that can propagate through the line. Under nonlinear operation, the Bragg frequency can be estimated as $f_{B}=1 /\left(\pi C_{a v} L\right)$, where $C_{a v}$ is the average capacitance of the varactor diode under a periodic excitation and $L$ the inductance value per cell. The NLTL used here is based on the hyper-abrupt varactor SMV1235 and for this separate design it has been excited with sinusoidal signals in the order of 100 MHz. Testing several combinations of varactor bias voltage $V_{b}$ and inductance $L$, the highest spectral content is obtained with $L=0.2 \mathrm{nH}$ and $V_{b}=-0.6 \mathrm{~V}$. The selected number of cells is $n=8$, which enables a compact design without significant degradation due to the diode resistive loss. The simulated output spectrum when terminating the line in its average characteristic impedance $Z_{c}=\sqrt{L / C_{a v}}$ is shown in Fig. 2. The high harmonic generation capabilities of this design can be noted.

Next the NLTL is introduced into the circuit of Fig. 1(a), replacing the bias voltage source, which gives rise to the prototype in Fig. 1(b). The photograph is shown in Fig. 1(c). When driven with the low frequency sinusoidal input signal, the NLTL output should be able to switch the oscillation on and off, in a manner similar to the pulse injection locked oscillators in [5-7]. The voltage waveforms obtained at the transistor base terminal for different values of the inductance $L$ are shown in Fig. 3. For low $L$, the output pulse delivered by the NLTL has too small duty cycle and the oscillation is on for a very small fraction of the period. As $L$ increases, the duty cycle increases too and for too large $L$ values, the NLTL output signal is no longer a pulse which explains the behaviour in Fig. 3(d).
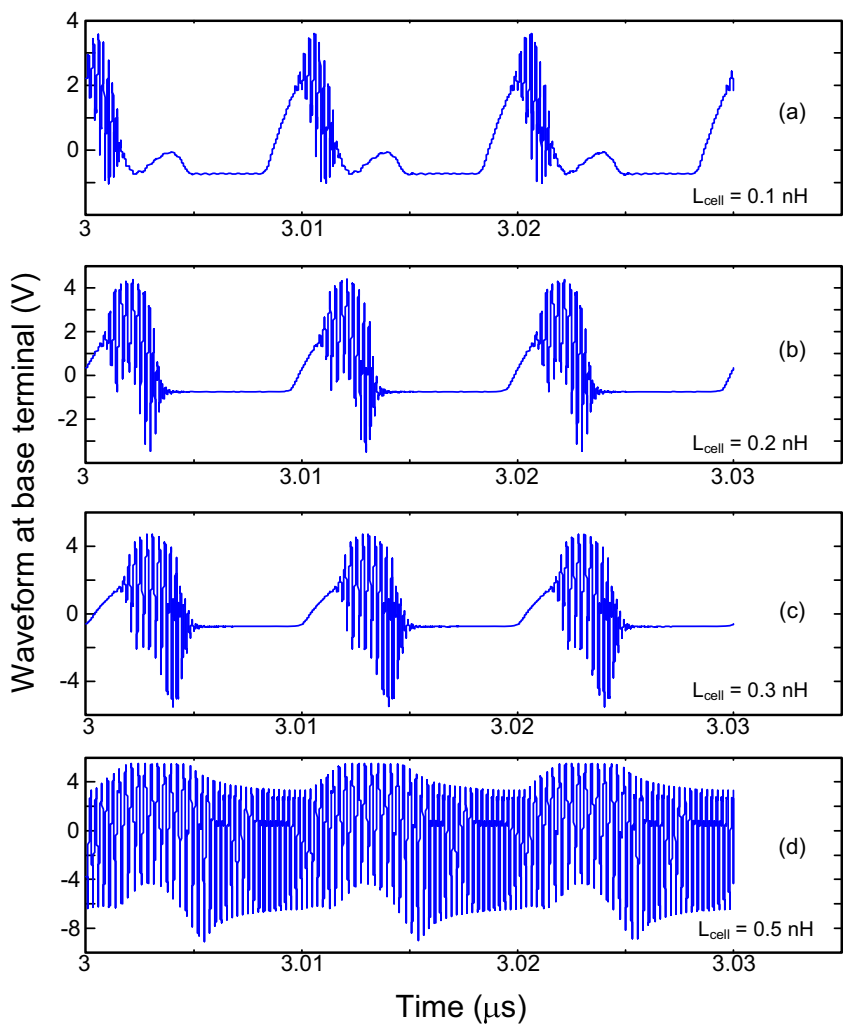

Fig. 3 Subharmonic injection-locked oscillator. Waveforms at the intrinsic base terminal for different values of cell-inductance, when the NLTL is excited with a signal of amplitude $E_{\text {in }}=1 \mathrm{~V}$ and frequency $f_{\text {in }}=100 \mathrm{MHz}$. 


\section{STABILITY ANALYSIS OF THE SUB-HARMONIC INJECTION-LOCKED OSCILLATOR}

The harmonic-balance (HB) analysis of the synchronization bands of a high order subharmonic injection-locked oscillator is cumbersome, especially in the case of an oscillation switched on and off. This is due the high number of harmonic terms required and the multiple nonlinear resonances generated in the system, associated to points of infinite slope in the solution curves, which prevent a direct tracing of these curves in commercial HB. Instead, the Poincaré map technique $[5,8]$ will be used for an analysis of the oscillator synchronization ranges. The map is obtained through the detection of the oscillation burst maxima once the circuit has reached the steady state. In the case of a solution of the same period as the input signal (desired subharmonic synchronization) all the maxima will have the same amplitude and a single point will be provided by the map. In the case of a period-doubled solution (frequency division by two) the map will provide two points, and in the general case of a frequency division by $\mathrm{n}$, it will provide $\mathrm{n}$ points. When the steady-state solution is quasi-periodic or chaotic, the map provides a large distribution of points, corresponding to maxima of different values.

The described map has been calculated versus the frequency $f_{i n}$ of the input sinusoidal signal of constant amplitude $E_{\text {in }}=1 \mathrm{~V}$. Fig. 4 presents the maps obtained in the lower and upper ranges of input frequencies. As can be seen in Fig. 4(a), the oscillator operates in the desired subhramonic injection-locked regime for some frequency intervals, through which the map provides only one point. These intervals are delimited by two different phenomena: flip bifurcations, leading to a frequency division by two (two points), and Hopf bifurcations, leading to a quasi-periodic regime (distribution of points). Note that the onset of a quasi-periodic regime in a secondary Hopf bifurcation is one of the fundamental mechanisms of desynchronization [8]. Fig. 4(b) shows the map corresponding to the upper input frequency range, where the same instability mechanisms (flip and Hopf bifurcations) are observed. In both cases [Fig.4(a) and Fig. 4(b)] and despite the low voltage value, there are distinct frequency intervals exhibiting the desired sub-synchronized behavior. However, as expected, broader subsynchorization bands are obtained for higher input frequency. Note that in Fig. 4(b) this input frequency is more than ten times lower than the original oscillation frequency.

Measurement results corresponding to the input frequency range of Fig. 4(a) are presented in Fig. 5 and Fig. 6. Fig. 5(a) shows the pulsed-envelope oscillation waveform obtained at $f_{\text {in }}=87.1 \mathrm{MHz}$, where the map accurately predicts the desired subharmonic behavior. The periodic spectrum with $87.1 \mathrm{MHz}$ as fundamental frequency is shown in Fig. 5(b). Examples of measured period doubled and quasi-periodic solutions are shown in Fig. 6(a) and Fig. 6(b), respectively. The Poincaré map in Fig. 4(a) provides a good estimation of the overall circuit behavior. Small discrepancies with the measured results are attributed to inaccuracies in the models of the active and passive elements of the distributed prototype.

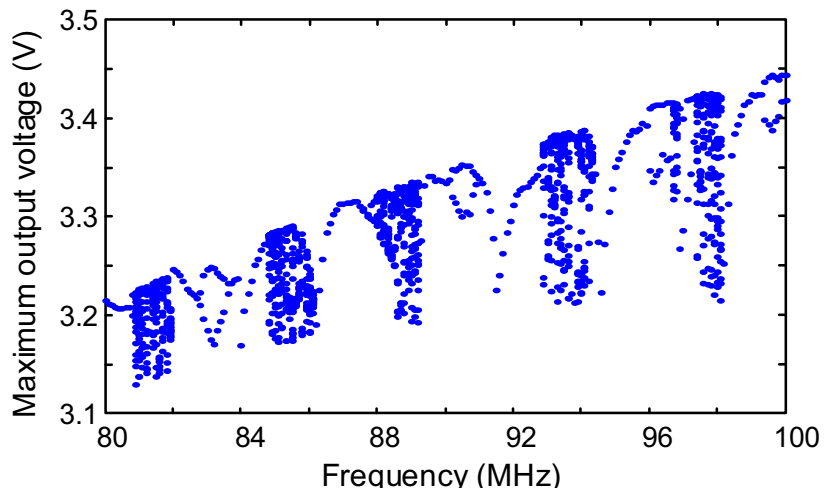

(a)

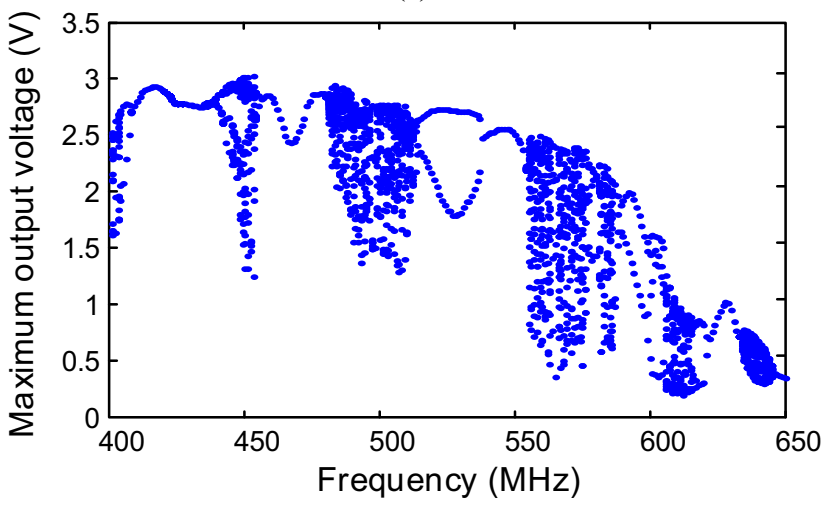

(b)

Fig. 4 Poincaré maps of oscillation-burst maxima applied to the sub-injection locked oscillator of Fig. 1(b), traced versus the input frequency at $E_{\text {in }}=1 \mathrm{~V}$. (a) Operation in the lower input frequency range $80-100 \mathrm{MHz}$. (b) Operation in the upper frequency range $400-650 \mathrm{MHz}$.

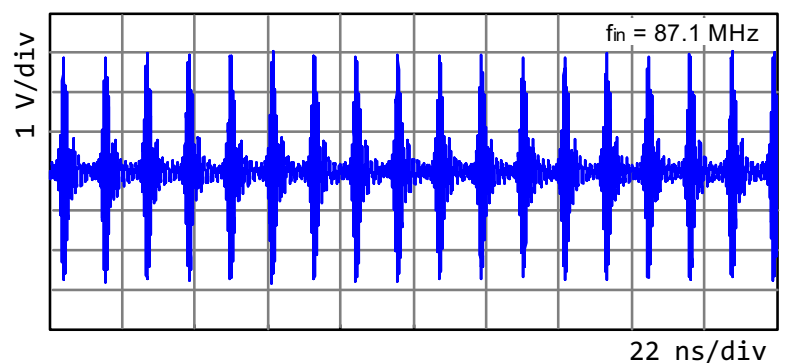

(a)

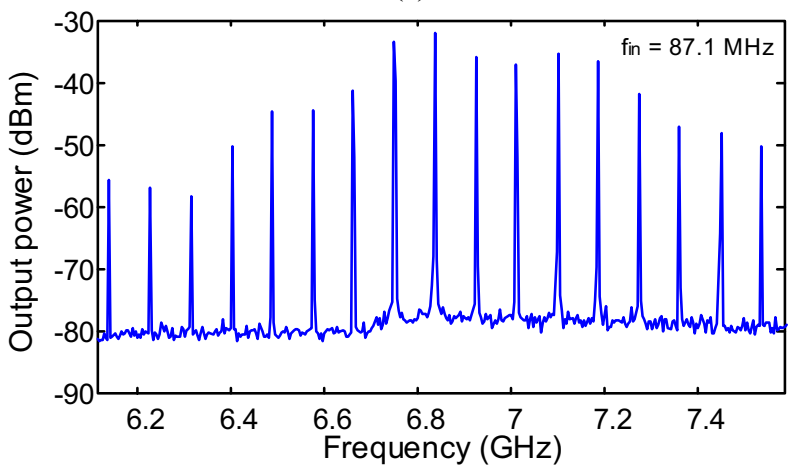

(b)

Fig. 5 Measured periodic solution of the subharmonic injection-locked oscillator when driven with $f_{\text {in }}=87.1 \mathrm{MHz}$ and $P_{\text {in }}=12.6 \mathrm{dBm}$. (a) Oscillation burst. (b) Spectrum. 


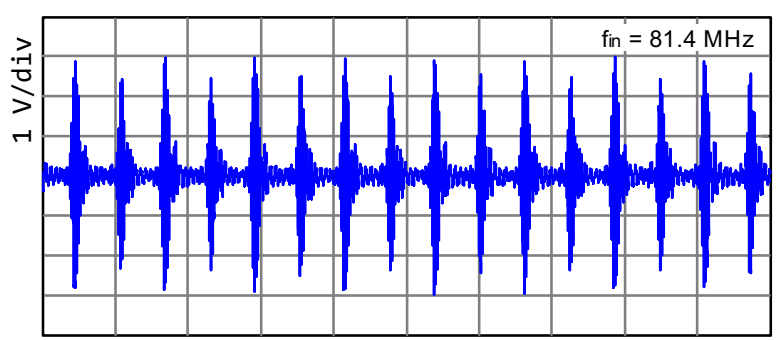

$10 \mathrm{~ns} / \mathrm{div}$

(a)

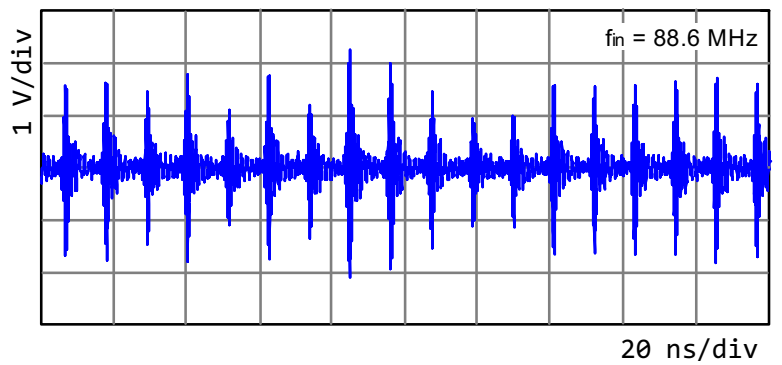

(b)

Fig. 6 Examples of measured solutions outside the synchronization ranges in Fig. 4(a). (a) Period-doubled solution at $f_{\text {in }}=81.4 \mathrm{MHz}$. (b) Quasi-periodic solution at $f_{\text {in }}=88.6 \mathrm{MHz}$.

\section{PHASE-NOISE ANALYSIS}

The NLTL based subharmonic injection-locked oscillator will have noise contributions from the low frequency injection signal and internal noise contributions, dominated by the transistor and varactor noise sources. The phase-noise spectrum of the subinjection-locked oscillator should follow the input phase noise, increased by $20 \log N$, where $N$ is the subharmonic order, up to certain corner frequency $f_{c}$. In general, this corner frequency is larger for a broader synchronization bandwidth. This behavior has been verified in simulation, by using a relatively high input frequency $f_{i n}$, which enables a HB simulation of the circuit and, therefore, the application of the conversion-matrix approach [9,10], which is the most accurate phase-noise analysis method when dealing with synchronized circuits. In Fig. 7(a), the resulting phase noise spectrum is compared with that of the input source. The input frequency is $477 \mathrm{MHz}$, which corresponds to a subharmonic order $N=15$ and, as can be seen, the output phase-noise spectrum follows the input phase noise, increased by $20 \log N$ up to corner frequency $f_{c}=200 \mathrm{kHz}$ [Fig. 7(a)]. The same analysis has been performed with experimental measurements. The measured phase-noise spectra of the input source and the subharmonic injection locked oscillators are shown in Fig. 7(b). The oscillator spectrum follows the input phase noise spectrum up to a similar corner frequency.

\section{ACKNOWLEDGMENT}

Spanish project TEC2011-29264-C03-01 for financial support.

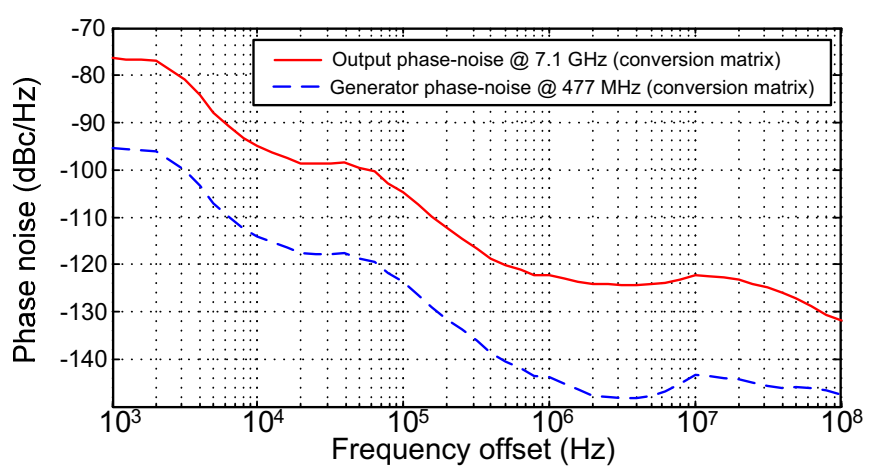

(a)

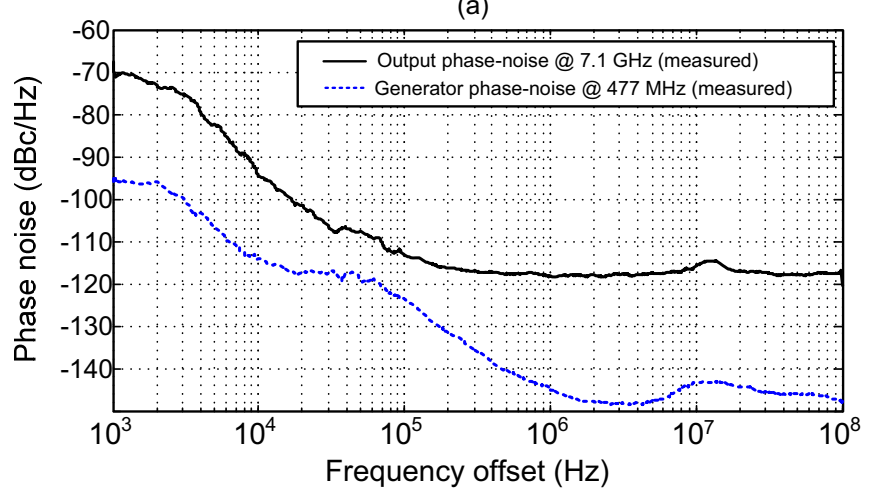

(b)

Fig. 7 Phase-noise analysis of the oscillator in Fig.1. Comparison of the phase-noise spectrum of the output signal and the input source. (a) Simulation with the conversion matrix approach. (b) Measurements.

\section{REFERENCES}

[1] Z. Xiangdong, X. Zhou, A.S. Daryoush, "A theoretical and experimental study of the noise behavior of subharmonically injection locked local oscillators," IEEE Trans. Microw. Theory and Techn., vol.40, no.5, pp.895-902, May, 1992.

[2] E. Afshari, A. Hajimiri, "Nonlinear Transmission Lines for Pulse Shaping in Silicon", IEEE J. Solid-State Circ., vol. 40, no.3, pp. 744$752,2005$.

[3] M. Rodwell et al. "GaAs Nonlinear transmission lines for picosecond pulse generation and millimetre wave sampling," IEEE Trans. Microw. Theory and Techn, vol. 39, no. 7, pp. 1194-2004, 1991.

[4] F. Martín, X. Oriols, J. A. Gil, J. García-García, "Optimization of Nonlinear Transmission Lines for Harmonic Generation", Int.. Jour. of Infrared and Millimeter Waves, Vol. 23, no. 1, pp 95-103, Jan., 2002.

[5] E. Fernandez; F. Ramirez ; A. Suarez; S. Sancho, "Stability and PhaseNoise Analysis of Pulsed Injection-Locked Oscillators," IEEE Trans. Microw. Theory and Techn, vol.61, no.1, pp.482-491, Jan., 2013.

[6] N. Deparis, C. Loyez, N. Rolland, P.A. Rolland, "UWB in millimetrc wave band with pulsed ILO”, IEEE Trans. Circuits and Systems II, vol. 55, no. 4, pp. 339-343.

[7] N. Deparis, A. Siligaris, P. Vincent, N. Rolland, “A 2 pJ/bit pulsed ILO UWB transmitter at $60 \mathrm{GHz}$ in 65-nm CMOS-SOI," IEEE International Conference on Ultrawideband, pp. 113-117, Oct., 2009.

[8] S. Parker, L. Chua, Practical Numerical Algorithms for Chaotic Systems, Springer, 1989.

[9] V. Rizzoli, F. Mastri, D. Masotti, "General noise analysis of nonlinear microwave circuits by the piecewise harmonic-balance technique IEEE Trans. Microw. Theory and Techn, vol. 42, no. 5, pp. 807-819, May, 1994.

[10] J.-C. Nallatamby, M. Prigent, M. Camiade, A. Sion, C. Gourdon, and J.J. Obregon, "An advanced low-frequency noise model of GaInP-GaAs HBT for accurate prediction of phase noise in oscillators", IEEE Trans. Microw. Theory and Techn, vol. 53, no. 5, pp. 1601 - 1612, May, 2005. 